\title{
Objetivos, obstáculos y riesgos de la formación científica del profesional
}

\author{
Miguel D. Rojo González
}

\section{Objetivos}

La actividad profesional tiene un carácter tecnológico en tanto consiste en la transformación de las manifestaciones de la realidad. El médico, el psicólogo, el ingeniero, y cualquier otro profesional están interesados, sobre todo, en las operaciones más eficientes para preservar o restablecer la salud física o mental, o para desviar el curso de un río o levantar un edifício, o para obtener, cualquier otro resultado apetecido, en un área particular de la actividad humana.

Por ello el conocimiento profesional incluye, fundamentalmente, los "procedimientos" y las "reglas" (las técnicas) que regulan las acciones pertinentes para la obtención de determinados fines útiles al hombre.La actividad científica, por su parte, consiste en la busqueda del conocimiento de la realidad tal y como es. El biólogo, el físico, el sociólogo, o el investigador de la psicología, o cualquier otro científico, está interesado en conocer cada vez mejor el objeto de "su" ciencia tal y como es y no en elaborar procedimientos que permitan modificarlo. Por ello la actividad científica tiene como resultado la elaboración de descripciones y explicaciones de la realidad, que se formulan como "leyes", "principios", "datos", etc. que son

* Doutor, Departamento de Formación Básica de la Facultad de Psicologia de la Universidad de La Habana, Cuba. 
(o aspiran a ser) "verdaderos" y que expresan el objetivo científico de conocer la realidad en su esencia necesaria.

Luego, si el conocimiento tecnológico está orientado hacia la acción "práctico", "utilitaria", para la modificación de las manifestaciones de la realidad, mientras que la acción científica está orientada a la obtención de una representación "cognoscitiva", consistente en la descripción o la explicación de lo que hay de más estable y esencial en ella, tenemos que preguntarnos a que fin sirve la formación científica del profesional?

Está claro que habrá tantas respuestas a esta pregunta como concepciones diferentes del profesional y de la profesionalidad. Aquellos para quienes el profesional se mide por el arsenal de sus "recetas" o "esquemas" para resolver problemas, responderán que su formación científica es practicamente innecesaria, los demás darán diversas respuestas en función de la relación que establezcan entre la formación científica y las cualidades deseables del profesional. Es a esto último a lo que quiero referirme.

a) A pesar de esta diferencia que acabo de señalar en cuanto a sus objetivos, la ciencia y la tecnología guardan entre si estrechas relaciones en primer lugar de carácter lógico. "Lógicamente" el conocimiento científico sirve de "fundamento" a los procedimientos y las reglas tecnológicas. Según Bunge (1971, p. 696) “... es más fácil predicar la fundamentación de las reglas que decir exactamente en qué consisten esos fundamentos", no obstante lo cual esta relación es reconocida por todos o la mayoría de los autores.Sin pretender sentar cátedra respecto a esta cuestión, al parecer espinosa, de la filosofía de la tecnología, pudiéramos decir que el fundamento de una regla o de un procedimiento tecnológico es el conocimiento científico en tanto este "explica" o "justifica" su éxito. Una regla o un procedimiento son "viables" cuando producen los resultados apetecidos y están "fundamentados" cuando ese efecto que producen se "explica", se "justifica", se "fundamenta" en el conocimiento científico de los objetos sobre los que se ha actuado. A los efectos de ejemplificar esto que acabo de decir supongamos que según una regla de la dietética "para prevenir trastornos digestivos no debemos ingerir carbohidratos acompañando a las proteínas" ... supongamos también que esta regla hubiera sido seguida por multitud de personas con muy buenos resultados. Entonces pudiéramos decir que la regla es "viable" o "efectiva", pero sólo diremos que está "fundamentada" cuando seamos capaces de explicar su efecto positivo, para lo cual tendremos que apoyarnos, necesariamente, enel conocimiento científico de la digestión.Ahora bien, ¿en qué sentido el conocimiento de los fundamentos de las reglas puede aumentar la capacidad o la eficiencia del profesional?, ¿ no sería suficiente el conocimiento de la norma y la probabilidad de susefectos para la formación del profesional?. No, y ello por varias razones entre las cuales, al menos las siguientes: una, que el conocimiento del fundamento permite evaluar la regla anticipadamente, prever sus efectos y evitar el costo que supone su aplicación "experimental"; dos, que este conocimiento 
permite una aplicación más flexible y precisa de la regla en cuanto hace posible tener en cuenta condiciones alternativas en cada caso particular; y tres, que el conocimiento de los fundamentos permite la generación de hipótesis, una observación más rica y profunda de los efectos de las reglas y los procedimientos y con ello una productividad mucho mayor del profesional. Luego el conocimiento de los fundamentos científicos de las normas y los procedimientos es valioso para el profesional, en primer lugar por que mediante ellos aumenta su eficiencia al aumentar su capacidad de prevenir con la más alta probabilidad los efectos de su aplicación; en segundo lugar por que este conocimiento le permite una mayor flexibilidad y precisión en la aplicación de las reglas y los procedimientos y, en tercer lugar, por que sobre la base de los fundamentos científicos puede realizar una observación más rica de los efectos de las reglas y los procedimientos y generar hipótesis que le permitan, incluso, perfeccionarlos.

b) La formación científica del profesional es valiosa también en tanto permite luchar contra el riesgo de la "tecnocratización". Permítaseme detenerme un instante en la distinción entre "tecnológico" y "tecnocrático", por que, en alguna ocasión, encontré resistencias, que no quisiera ver repetidas, generadas en la confusión de estos dos términos.Hay que decir, en primer lugar, que no todo lo "tecnológico" es "tecnocrático" y que "tecnológico" no tiene ningún contenido valorativo mientras que "tecnocrático", sí. Todo profesional es un "tecnólogo" en la medida en que se especializa en la aplicación de un conjunto de procedimientos, reglas y normas que forman una "tecnología"; pero solo algunos son "tecnocratas" en la medida en que hiperbolizan los criterios técnicos y que se juzgan pertenecientes a una clase o casta superior al resto de los mortales. Aspiramos, por supuesto, a que los profesionales sean "tecnólogos" pero no "tecnócratas". Como lograrlo? Como evitar el riesgo de la "tecnocratización"? Creo que hay múltiples maneras de enfrentar este riesgo pero me voy a referir aquí a la formación científica como una de ellas.Como acabo de señalar, la actitud tecnocrática consiste en una hiperbolización, en una exageración de la importancia o el valor de un conjunto de conocimientos y de criterios, es, por tanto, una visiónunilateral que toma en cuenta sólo algunos elementos olvidando el resto.Nada más alejado de la formación cientíca cuando esta se entiende también como inculcación de la "cultura científica". En efecto, la formación científica puede ser entendida, al menos de dos formas; una, como simpletransmisión de los conocimientos y los procedimientos propios de una ciencia y dos, como inculcación o facilitación de la apropiación de la cultura científica, además de lo anterior.La formación científica puede resultar en un reforzamiento de la actitud tecnocrática cuando ella se entiende solamente como "fundamentación" de la técnica. Para la soberbia y la altaneria tecnocráticas la fundamentación científica puede ser un argumento adicional. Para que la formación científica sirva a los efectos de formar un profesional y no un tecnócrata ha de entenderse también como "inculcación de la cultura científica" para de este modo compensar, balancear, las tendencias eficientistas, utilitaristas, economicistas que la formación técnica puede estimular.La cultura científica se desprende de la formación científica cuando ésta no se limita a la enseñanza de repertorios 
de conocimientos y de métodos sino que consiste también en la transmisión del conjunto de concepciones, de criterios y de valores directamente ligados a la actividad científica, a la concepción científica del mundo y de las relaciones entre sus partes, a la concepción de la ciencia y del hombre.Luego, otro de los objetivos de la formación científica del profesional puede ser el de evitar el riesgo de la "tecnocratización" inculcando una "cultura científica" compuesta por las concepciones, los criterios y losvalores que se desprenden del conocimiento científico del mundo y de la actividad cognoscitiva de los hombres a lo largo de su historia.

c) La formación científica entendida como la inculcación de la cultura científica tiene otra consecuencia importante para la formación del profesional y es la de su formación como ciudadano. En cualquiera de los "mundos", primero, segundo o tercero, pero aún más en el caso de este último, la formación del profesional sirve al propósito del progreso y del desarrollo. Si los países invierten dinero en el financiamiento de las universidades para la formación de los profesionales es, en el caso de algunos, para mantener su supremacía mundial en los campos de la ciencia y la técnica y en el caso de otros para formar "el capital humano" 1 con cuyas "rentas" llegar al tan ansiado "desarrollo". Es evidente que el desarrollo consiste en una transformación y siendo los profesionales la clase social con el mayor potencial técnico de acción transformadora llevan sobre sus hombros gran parte de la responsabilidadpor el desarrollo de la sociedad.Ahora bien, desde el punto de vista de la formación del individuo el desarrollo consiste en "...el mejoramiento de sus capacidades de elección" (Michalon, 1996, p. 20) es decir, del mejoramiento, en primer lugar, delgrado de responsabilidad con que asumen sus elecciones, en segundo lugar de los criterios con que eligen y en tercer lugar, de su dominio del procedimiento, directamente ligado a la toma de decisiones, que consiste enexaminar o sopesar información para llegar a conclusiones. Y, precisamente, no hay ocasión mejor para propiciar la adquisición de estas cualidades, criterios y capacidades que la actividad ligada a la formación científica si ésta no se limita a transmitir un saber y cumple verdaderamente su funciónde propiciar la apropiación de la cultura científica. Éste es el modo en quela formación científica puede hacer su más valiosa contribución a laeducación del ciudadano.

\section{Obstáculos}

Un tipo de obstáculo tiene que ver con los intereses de los estudiantes.Sabemos que la "relevancia" es el criterio fundamental con que losestudiantes juzgan cualquier contenido incluído en los planes de estudio 
ysabemos también que, para la mayor parte de ellos, solo son "relevantes" loscontenidos relativos al "saber hacer", es decir, las "normas", las "reglas" y los "procedimientos" directamente relacionados con el ejercicio de laprofesión. No es extraño que así sea pues hoy día los empleos son cada vezmás escasos, la rivalidad por cada puesto de trabajo es cada vez másenconada y los empleadores perfeccionan cada vez más los métodos paradetectar y elegir a los más "eficientes". En estas condiciones la búsquedade la "competitividad" suele ser la motivación fundamental para el ingresoen los centros de educación superior.Esto trae como consecuencia el desinterés de los estudiantes por losconocimientos para los que no encuentra una "utilidad inmediata" y, enconsecuencia, la hiperbolización de los criterios tecnocráticos relacionadoscon la "viabilidad" y la "eficiencia" y el menosprecio de los criterios de "verdad", "objetividad" y "fundamentación" propios de la culturacientífica. El joven se inclina así, inconsciente e involuntariamente, dellado de la formación tecnocrática. Esta situación resulta un verdaderoobstáculo para la formación de la base científica de los conocimientosprofesionales así como para la facilitación de la apropiación de la culturacientífico-técnica.

Éste es un obstáculo difícil de remover en tanto, como hemos dicho, parece congruente con el espíritu que domina hoy el mercado laboral, peroademás resulta, reforzado involuntariamente, por la estructura curricularde los planes de formación profesional que, generalmente, agrupan laformación "científica básica" en los primeros años de las carrerasprolongando de este modo, al inicio del nivel superior, la duración delnivel de la enseñanza general, preuniversitaria, e impacientando a losestudiantes que llegan con la ilusión de ponerse en contacto, de inmediato,con el "saber hacer" profesional.En mi opinión, para enfrentar este obstáculohay que comenzar por revisar el concepto de "conocimiento básico" y, sobretodo, la relación entre "básico" y "precedente" o "previo". En efecto segúnuna hipótesis implícita y nunca suficientemente demostrada los conocimientosque consideramos "básicos" deben ser ubicados, necesariamente, al inicio dela formación profesional. Procedemos así por analogía con los ingenieros y los arquitectos que nunca levantan una pared sin poner "antes" sus "bases“.¿Pero, es valida esta analogía en el caso de la adquisición del saber? En miopinión, no. El carácter básico, precedente, de la formación científica en relación conla adquisición del "saber hacer" profesional estriba en que se considera que ellapuede servir como "anclaje" de éste. Pero ésta no es, en realidad, sufunción más importante. En realidad el aprendizaje de una técnica norequiere de la asimilación de un conjunto de "conceptos", "principios" y "leyes" como los que 
componen el conocimiento científico cualquier individuo puede aprender a aplicar las normas de cualquier tecnología sin "necesidad" del conocimiento científico que las fundamenta (y esto es lo quesucede en el caso de la formación de técnicos-medio y operarios, p.e.). Laverdadera función del conocimiento científico-básico, como he dicho antes,no es entonces la de servir de "anclaje", sino la de ampliar, profundizar, flexibilizar y potenciar el "saber hacer" profesional. Por ello, en miopinión, no es necesario ubicarlo en los años iniciales del período deformación profesional y puede ser situado en el momento en que resulte másoportuno y en el que su impartición revista mayor significación o relevanciapara el estudiante.

b) Otro tipo de "obstáculo" es de carácter cognoscitivo o epistemológico ytiene que ver con estructuras y/o representaciones mentales que estorban laasimilación o la apropiación de otros contenidos con los que no soncongruentes o coherentes (ver Bachelard, G. 1974).Se trata de los "conceptos" y de la "cultura" del estudiante que ingresa enlos centros de formación profesional. Este obstáculo no es igualmenteimportante en todas las carreras - en unas resulta más significativo queen otras. La diferencia depende de la distancia entre el aparato conceptualy las acciones científico-profesionales y el vocabulario y las accionescotidianas. Cuánto menor esta distancia tanto mayor suele ser el obstáculo. En su vida cotidiana y a través de sus años de escolarización el estudianteadquiere o se apropía, espontánea o voluntariamente, de muchos conceptos,concepciones, criterios y valores referidos a la ciencia y la tecnología queresultan luego verdaderos obstáculos epistemológicos, cognoscitivos, en suformación profesional.En relación con la formación científica el estudiante tiene muchas veces una "cultura científica" compuesta por concepciones, criterios y valores nadadeseables. La ciencia aparece como "difícil", "poco útil" , "esotérica" etc., y la verdad, la claridad, la objetividad, la precisión, la coherenciaetc., no son valores del pensamiento, del conocimiento ni de la expresión; nose conoce la función de la ciencia ni la de la tecnología, ni susrelaciones, no se concibe el mundo como un sistema ordenado, la noción decausalidad o de determinación universal está poco formada, etc. Todos estosson obstáculos que deben ser removidos si queremos no solo dar unainformación científica sino también formar la cultura científica de la quevengo hablando.Hay que decir que la remoción de obstáculos, cognoscitivos oepistemológicos, no tiene que ser la tarea del profesor. Frecuentemente latarea del profesor se concibe como la de un "facilitador" y esto se entiendeen el sentido de que su función consiste en eliminar o en minimizar losobstáculos que el estudiante puede encontrar para la comprensión de unconcepto, la aplicación de una técnica o la solución de un 
problema. En miopinión esto es nocivo. El aprendizaje mejor ocurre cuando el estudiantevence obstáculos "a su medida" y la tarea del profesor no consiste enremover todos los obstáculos, sino en "medir" el obstáculo para determinarcuál debe vencer el estudiante y cuál es el que el debe remover.

\section{Riesgos}

Antes hablé de la "tecnocratización" ahora quiero referirme a los que, en miopinión, son dos de los mayores riesgos de la formación científica delprofesional : la "dogmatización" y la "esquematización". La "dogmatización" tiene que ver con la formación de "escuelas", con laconformación del pensamiento del estudiante en una sola dirección, con laeliminación de su espíritu crítico y de su libertad de discernimiento yelección. Debido a que la formación científica ocurre, generalmente, en losprimeros años de las carreras universitarias, o sea en el momento en que losestudiantes son más receptivos y están más abiertos a las concepciones, criterios y valoraciones que reciben es muy fácil producir en ellos unaadhesión "dogmática" a una posición científica dada.Este dogma no se refiere muchas veces sólo a una concepción teórica ometodológica dentro de una ciencia particular sino que se refiere tambiénal propio concepto de ciencia. El estudiante adopta de forma rígidadeterminadas concepciones respecto de "lo científico" y "lo no científico" que se convierten en "obstáculos" para la asimilación de contenidossiguientes en sus estudios.La "esquematización" puede manifestarse de muchos modos pero quizás es másevidente en el caso de las llamadas "competencias investigativas". Aunque nolo dije antes, en muchas ocasiones, un objetivo de la formación científica espreparar al estudiante para realizar investigaciones, que es una tareafrecuente de algunos profesionales, y que incluso sirve para preparar lastésis con las que frecuentemente culminan los estudios. El concepto de "competencias investigativas" es polivalente; se puede referir a lashabilidades, a las destrezas, a las capacidades, etc. necesarias pararealizar la actividad investigativa. Frecuentemente lo que se entiende poreste concepto es un conjunto de operaciones (tales como "formular unproblema", "formular una hipótesis", etc.) que se ejecutan en un ordenrelativamente constante. La estrategia formativa se desarrolla frecuentemente como sigue: el 
profesor determina el conjunto de lascompetencias investigativas y el orden o secuencia en el que intervienen enla investigación, diseña las situaciones correspondientes y proveesuficientes ocasiones de ejercitación. Lo que se logra en muchas másocasiones que las deseables es producir una cierta rigidez, unainflexibilidad del pensamiento... no se forman realmente "competenciasinvestigativas" ... el estudiante "memoriza" un esquema que trata de aplicartal cual a todas las situaciones que "le parecen" pero que no siempre sonsemejantes. Como se ve estos dos riesgos se asemejan en tanto dependen (o crean) unacierta rigidez del pensamiento, una incapacidad para aceptar alternativas, para adecuarse a situaciones nuevas y diferentes y son, por ello, completamente incompatibles con la "cultura científica" que debecaracterizarse, precisamente, por una "liberación" del espíritu y una "potenciación" de la inteligencia de los hombres.En mi opinión la evaluación de la necesidad o la importancia de la formacióncientífica del profesional requiere una reflexión sobre el concepto de "profesional". Al respecto considero, en primer lugar, que la capacidadprofesional no consiste solo en la posibilidad de "aplicar" con precisión yeficiencia el conjunto de procedimientos y normas propios de una profesióndada, sino también en la posibilidad de producir, transformar, adecuar,perfeccionar, etc. dichos procedimientos y técnicas, en segundo lugar, quelas actitudes y las concepciones tecnocráticas son indeseables y en tercerlugar, que dada su potencia transformadora la responsabilidad social de losprofesionales es muy alta por lo que su formación ciudadana reviste una importancia fundamental. Tomando como meta esta representación de laprofesionalidad la formación científica resulta del mayor interés educativosiempre y cuando no se la entienda sólo como la transmisión de un saber y deun saber hacer sino también como la facilitación de la apropiación de lacultura científica. A este proceso de inculcación y apropiación de lacultura dedicaré un artículo próximo.

\section{RESUMEN}

En este artículo el autor expone sus opiniones, fruto de más de 25 años dedicado a la formación científica de profesionales universitários, sobre los objetivos, los obstáculos y los riesgos de la formación científica de los profesionales. Considera así que la formación científica puede contribuir significativamente a la eficiencia y la productividad del profesional en 
tanto le brinda el fundamento del saber tecnológico y com ello la posibilidad de crear nuevas reglas e instrumentos y de valorar anticipadamente sus resultados posibles. Entre los obstáculos se señala, en primer lugar, la falta de coincidencia entre la orientación práctica-profesional con que llegan los estudiantes a la universidad y lo que los currículos le ofrecen en ese momento y, en segundo lugar, las concepciones "científicas" adquiridas espontáneamente en la vida cotidiana.Por último el autor nos habla de como a través de la formación científica se puede combatir y minimizar dos de los mayores riesgos de la formación tecnológica, como son, la dogmatización y la tecnocratización.

\section{BIBLIOGRAFÍA}

BACHELARD, Gaston. La formación del espíritu científico, 1974.

CONTRIBUICIÓN a un psicoanálisis del conocimiento objetivo. Siglo XXI, Argentina.

BUNGE, Mario. La investigación científica. Editorial de Ciencias Sociales. Cuba: Instituto Cubano del Libro, 1971.

MICHALON, Clair. Amelioration des capacites de choix. Diagonales. n. 40, Nov., Francia, 1996. 